\title{
Common Fixed Points of Faintly Compatible Mappings
}

\author{
Mintu Lal Saha', Saurabh Manro ${ }^{2}$ \\ ${ }^{1}$ Department of Mathematics, Handique Girls' College, Guwahati 781001,Assam, India. \\ ${ }^{2}$ School of Mathematics and Computer Applications , Thapar University, Patiala (Punjab), India.
}

Abstract: In this paper, we have established some common fixed point theorems for a pair of faintly compatible mappings by removing the containments and weakening the continuity conditions from the results due to Bisht and Shahzad [2]. We have used a weaker notion of reciprocal continuity and thereby obtaining a proper generalization of these results.

Keywords: Compatible maps, faintly compatible maps , reciprocal continuous maps , property E.A. , common fixed points.

\section{INTRODUCTION}

In recent years, the study of fixed point theorems satisfying some contractive-type conditions have been at the centre-stage of some intense research activity and a large number of research papers devoted to the development of fixed point theorems and their applications have appeared in the literature. To mention a few ,we cite $[3,4,5,6,12,13]$.

A common fixed point theorem is a statement containing a set of conditions sufficient to ensure the existence of a common fixed point of a number of self - maps in a metric space. Most of these theorems mainly contain a commutativity condition, a condition on the ranges of the maps, some continuity conditions and a contractive or possibly a Lipschitz-type or a Banach -type or a Boyd and Wong -type condition. Most of the authors used one or more of these conditions or their various forms and obtained some remarkable success thereby enriching the literature on fixed point theory. For instance, Jungck [7] introduced the notion of compatibility in the year 1986 by weakening the concept of weakly commuting maps introduced by Sessa [16] in the year 1982 and established some important common fixed point theorems in a series of his papers [7, 8, 9]. Pant [11] introduced the notion of $R$-weak commutativity of maps in metric spaces by generalizing the notion of commutativity in the year 1994 . In 1996 , Jungck [10] again generalized the notion of compatible maps by introducing weak compatible maps. Very recently , Bisht and Shahzad [2] introduced the notion of faintly compatible maps as those conditionally compatible maps which commute on a non empty set of coincidence points of a pair of self-maps $A$ and $S$ in a metric space and also established some interesting common fixed point theorems for non-commuting maps under both contractive and non-contractive conditions. It may be worth mentioning that the notions of faint compatibility and conditional compatibility are independent of each other and this was established by various examples in the paper [2].They further claimed that the faint compatibility is a necessary condition for the existence of a common fixed point of a pair of self-maps in a metric space.

In our present attempt, we have proved some common fixed point theorems involving faintly compatible, reciprocal continuous, non- commuting maps in a metric space. Our results generalize the theorems due to Bisht and Shahzad [2] in the sense that these results eliminate the containment conditions and also use weaker continuity conditions and thereby obtaining proper generalizations. We further illustrate our results by suitable examples.

\section{Preliminaries}

In this section, we recall some definitions and useful results which are already in the literature.

Definition 2.1. A pair of self-maps $[A, S]$ of a metric space $(X, d)$ is said to be

(a) compatible [7], if $\lim _{n \rightarrow \infty} d\left(A S x_{n}, S A x_{n}\right)=0$, whenever $\left\{x_{n}\right\}$ is a sequence in $X$ such that $\lim _{n \rightarrow \infty} A x_{n}=$ $\lim _{n \rightarrow \infty} S x_{n}=x$, for some $x \in X$.

(b) non-compatible, if $[A, S]$ is not compatible, i.e., if there exists a sequence $\left\{x_{n}\right\}$ in $X$ such that $\lim _{n \rightarrow \infty} A x_{n}=$ $\lim _{n \rightarrow \infty} S x_{n}=x$, for some $x \in X$, and $\lim _{n \rightarrow \infty} d\left(A S x_{n}, S A x_{n}\right)$ is either non-zero or non-existent.

${ }^{2}$ Corresponding Author: sauravmanro@hotmail.com 
(c) Weak compatible [10], if the pair commutes on the set of their coincidence points, i.e. , for $x \in X, A x=S x$ implies $A S x=S A x$.

(d) conditionally compatible [15] , if whenever the set of sequences $\left\{x_{n}\right\}$ satisfying $\lim _{n \rightarrow \infty} A x_{n}=\lim _{n \rightarrow \infty} S x_{n}$ is non-empty, there exists a sequence $\left\{z_{n}\right\}$ in $X$ such that $\lim _{n \rightarrow \infty} A z_{n}=\lim _{n \rightarrow \infty} S z_{n}=t$, for some $t \in X$ and $\lim _{n \rightarrow \infty} d\left(A S z_{n}, S A z_{n}\right)=0$.

(e) Reciprocal continuous [14] , if $\lim _{n \rightarrow \infty} A S x_{n}=A x, \lim _{n \rightarrow \infty} S A x_{n}=S x$, whenever $\left\{x_{n}\right\}$ is a sequence in $X$ such that $\lim _{n \rightarrow \infty} A x_{n}=\lim _{n \rightarrow \infty} S x_{n}=x$, for some $x \in X$.

$(f)$ Faintly compatible [2] , if $[A, S]$ is conditionally compatible and $A$ and $S$ commute on a non-empty subset of the set of coincidence points, whenever the set of coincidence points is nonempty.

(g) Satisfy the property $\boldsymbol{E} . \boldsymbol{A}$. [1] , if there exists a sequence $\left\{x_{n}\right\}$ in $X$ such that $\lim _{n \rightarrow \infty} A x_{n}=\lim _{n \rightarrow \infty} S x_{n}=x$, for some $x \in X$.

The property E.A. was introduced by Aamri and Moutawakil [1] and is a generalization of the concept of non compatibility. The notions of faint compatibility and non-compatibility are independent concepts. Moreover , compatibility ensures faint compatibility but the converse is not true. The following example illustrates this fact.

Example 2.2. [2] Let $X=[3,6]$ and $d$ be the usual metric on $X$. Let $A, S$ be self maps of $X$ given by

$A x=3$, if $x=3$ or $>5, A x=x+1$, if $3<x \leq 5$.

$S 3=3, S x=\frac{x+7}{3}$, if $3<x \leq 5, S x=\frac{x+1}{2}$, if $x>5$.

Then $[A, S]$ is faintly compatible but not compatible.

Lemma 2.3. If $[A, S]$ is a pair of compatible self maps in a metric space $(X, d)$ then they are weak compatible. But the converse is not true.

Example 2.4. Let $X=[0,2]$ and $d$ be the usual metric on $X$. Let $A, S$ be self maps of $X$ given by

$$
A x=\left\{\begin{array}{c}
2-x, \text { if } 0 \leq x \leq 1 \\
2, \text { if } 1<x \leq 2
\end{array} \text { and } S x=\left\{\begin{array}{c}
x, \text { if } 0 \leq x \leq 1 \\
2, \text { if } 1<x \leq 2
\end{array}\right.\right.
$$

Then $[A, S]$ is not compatible but is weak compatible.

Lemma 2.5. [14] If $[A, S]$ is a pair of continuous self maps of a metric space $(X, d)$, then they are reciprocal continuous. But the converse is not true.

Example 2.6. [14] Let $X=[2,20]$ and $d$ be the usual metric on $X$. Let $[A, S]$ be a pair of self maps of $X$ given by

$A x=\left\{\begin{array}{l}2, \text { if } x=2 \\ 3, \text { if } x>2\end{array}\right.$ and $S x=\left\{\begin{array}{l}2, \text { if } x=2 \\ 6, \text { if } x>2\end{array}\right.$

Then $[A, S]$ is reciprocal continuous but not continuous.

\section{MAin ReSUlts}

Theorem 3.1. Let $A$ and $S$ be self maps of a metric space $(X, d)$ such that

(3.1.1) $[A, S]$ is non-compatible ,

(3.1.2) $[A, S]$ is faintly compatible ,

(3.1.3) $d(A x, A y) \leq k d(S x, S y)$, for every $x, y \in X$ and some $0 \leq k<1$,

(3.1.4) $[A, S]$ is reciprocal continuous.

Then $A$ and $S$ have a unique common fixed point.

Proof. Let $\left\{x_{n}\right\}$ be a sequence in $X$ such that $A x_{n} \rightarrow t, S x_{n} \rightarrow t$, for some $t \in X$ and $\lim _{n \rightarrow \infty} d\left(A S x_{n}, S A x_{n}\right)$ is either non-zero or non-existent (This is by virtue of (3.1.1)). Since $[A, S]$ is faintly compatible and $\lim _{n \rightarrow \infty} A x_{n}=$ $\lim _{n \rightarrow \infty} S x_{n}=t$, there is a sequence $\left\{z_{n}\right\}$ in $X$ satisfying $\lim _{n \rightarrow \infty} A z_{n}=\lim _{n \rightarrow \infty} S z_{n}=u$, for some $u \in X$ such that $\lim _{n \rightarrow \infty} d\left(A S z_{n}, S A z_{n}\right)=0$. As $[A, S]$ is reciprocal continuous, we get $\lim _{n \rightarrow \infty} A S z_{n}=A u, \lim _{n \rightarrow \infty} S A z_{n}=S u$ and so $A u=S u$. As $[A, S]$ is faintly compatible, we get $A S u=S A u$ and so $A A u=A S u=S A u=S S u$. If $A u \neq A A u$, 


\section{American Research Journal of Mathematics, Volume 1, Issue 2, April 2015 ISSN 2378-704X}

we get by (3.1.3), $d(A u, A A u) \leq k d(S u, S A u)=k d(A u, A A u)$, implying $k \geq 1$, a contradiction. So $A u=A A u=$ $S A u$ and $A u$ is a common fixed point of $A$ and $S$. For uniqueness, let $\alpha, \beta \in X$ such that $A \alpha=S \alpha=\alpha, A \beta=$ $S \beta=\beta$, we get $d(\alpha, \beta)=d(A \alpha, A \beta) \leq k d(S \alpha, S \beta)=k d(\alpha, \beta)$ and so $d(\alpha, \beta)=0$ as $0 \leq k<1$. Therefore ,$\alpha=\beta$ and hence the common fixed point is unique. (3)

The following example illustrates the Theorem (3.1)

Example 3.2. Let $X=[0,4]$ and $d$ be the usual metric on $X$. Let $A, S$ be the self-maps of $X$ given by $A x=1$, if $x \leq 1, A x=2$, if $x>1$. $S x=2-x$, if $x \leq 1, S x=4$, if $x>1$.

(i) Let $x_{n}=1-\frac{1}{n} \in X$. Now $A x_{n} \rightarrow 1, S x_{n} \rightarrow 1, A S x_{n} \rightarrow 2, S A x_{n} \rightarrow 1$ and so

$d\left(A S x_{n}, S A x_{n}\right) \nrightarrow 0$. Therefore,$[A, S]$ is non-compatible.

(ii) Let $x_{n}=1$. Now $A x_{n} \rightarrow 1, S x_{n} \rightarrow 1$ and $A S x_{n} \rightarrow 1, S A x_{n} \rightarrow 1$ and so $d\left(A S x_{n}, S A x_{n}\right) \rightarrow 0$. Therefore, $[A, S]$ is conditionally compatible. Also $A 1=S 1$ and $A S 1=S A 1$. Hence $[A, S]$ is faintly compatible.

(iii) For $x, y \leq 1, d(A x, A y)=0 \leq \frac{1}{2} d(S x, S y)=\frac{1}{2}|x-y|$.

For $x, y>1, d(A x, A y)=0 \leq \frac{1}{2} d(S x, S y)=0$.

For $x \leq 1, y>1, d(A x, A y)=1 \leq \frac{1}{2} d(S x, S y)=\frac{1}{2}|2+x|$.

For $x>1, y \leq 1, d(A x, A y)=1 \leq \frac{1}{2} d(S x, S y)=\frac{1}{2}|2+y|$.

Therefore, the condition (3.1.3) is satisfied with $\mathrm{k}=1 / 2$

(iv) Let $x_{n} \in X$ such that $A x_{n} \rightarrow z, S x_{n} \rightarrow z$ in $X$. Then $x_{n}=1=z$ and $A S x_{n} \rightarrow A z, S A x_{n} \rightarrow S z$. Therefore, $[A, S]$ is reciprocal continuous.

Therefore, all the conditions of the Theorem (3.1) are satisfied and we note that $1 \in X$ is the unique common fixed point of $A$ and $S$. (3)

Remark 3.3. Both the functions $A$ and $S$ of the above example are discontinuous and so do not satisfy the continuity condition of the Theorem (2.1) of Bisht and Shahzad [2]. Therefore, the Theorem (3.1) is a proper generalization of the Theorem (2.1) due to Bisht and Shahzad.

Theorem 3.4. Let $A$ and $S$ be self maps of a metric space $(X, d)$ satisfying the following conditions:

(3.4.1) $[A, S]$ is non-compatible ,

(3.4.2) $[A, S]$ is faintly compatible ,

(3.4.3) $d(A x, A y)<d(S x, S y)$, whenever $S x \neq S y, x, y \in X$,

(3.4.4) $[A, S]$ is reciprocal continuous.

Then $A$ and $S$ have a unique common fixed point.

Proof. In view of (3.4.1), we get a sequence $\left\{x_{n}\right\}$ in $X$ such that $A x_{n} \rightarrow t, S x_{n} \rightarrow t$, for some $t \in X$ and $\lim _{n \rightarrow \infty} d\left(A S x_{n}, S A x_{n}\right)$ is either non-zero or non-existent . As $[A, S]$ is faintly compatible, there is a sequence $\left\{z_{n}\right\}$ in $X$ satisfying $\lim _{n \rightarrow \infty} A z_{n}=\lim _{n \rightarrow \infty} S z_{n}=v$, for some $v \in X$ such that $\lim _{n \rightarrow \infty} d\left(A S z_{n}, S A z_{n}\right)=0$. By (3.4.4), we get $\lim _{n \rightarrow \infty} A S z_{n}=A v, \lim _{n \rightarrow \infty} S A z_{n}=S v$ and so $A v=S v$. Again as $[A, S]$ is faintly compatible, we get $A S v=$ $S A v$ and so $A A v=A S v=S A v=S S v$. If $A v \neq A A v$, i.e. , $S v \neq S A v$, we get by (3.4.3), $d(A v, A A v)<$ $d(S v, S A v)=d(A v, A A v)$, a contradiction. So $A v=A A v=S A v$ and $A v$ is a common fixed point of $A$ and $S$. Let $\alpha, \beta \in X$ such $A \alpha=S \alpha=\alpha, A \beta=S \beta=\beta$. If $\alpha \neq \beta$, we get $d(\alpha, \beta)=d(A \alpha, A \beta)<d(S \alpha, S \beta)=d(\alpha, \beta)$, a contradiction. Therefore, $\alpha=\beta$ and hence the common fixed point is unique. (3)

We now give an example to illustrate the Theorem (3.4). The example (3.2) also illustrates the Theorem (3.4).

Example 3.5. Let $X=[2, \infty[$ and $d$ be the usual metric on $X$. Let $A, S$ be self maps of $X$ given by $A x=2$, if $x=2, A x=6$, if $x>2$. $S x=2$, if $x=2, S x=x+4$, if $x>2$. 
(i) Let $x_{n}=2+\frac{1}{n} \in X$. Then $A x_{n} \rightarrow 6, S x_{n} \rightarrow 6$ and $A S x_{n} \rightarrow 6, S A x_{n} \rightarrow 10$. Therefore, $\left.d\left(A S x_{n}, S A x_{n}\right)\right) \rightarrow 0$ and so $[A, S]$ is non- compatible.

(ii) With $x_{n}=2 \in X$, we get $A x_{n} \rightarrow 2, S x_{n} \rightarrow 2$ and $A S x_{n} \rightarrow 2, S A x_{n} \rightarrow 2$ and sod $\left(A S x_{n}, S A x_{n}\right) \rightarrow 0$. Also $A, S$ commute at the only point of coincidence $2 \in X$.Therefore, $[A, S]$ is faintly compatible.

(iii) For $x=y=2, d(A x, A y)=0 \leq d(S x, S y)=0$.

For $x, y>2, d(A x, A y)=0 \leq d(S x, S y)=|x-y|$.

For $x=2, y>2, d(A x, A y)=4<d(S x, S y)=y+2$.

For $x>2, y=2, d(A x, A y)=4<d(S x, S y)=x+2$.

Therefore, the condition (3.4.3) is satisfied.

(iv) Let $x_{n} \in X$ such that $A x_{n} \rightarrow z, S x_{n} \rightarrow z$ in $X$. Then $x_{n}=2=z$ and $A S x_{n} \rightarrow A z, S A x_{n} \rightarrow S z$. Therefore, $[A, S]$ is reciprocal continuous.

Therefore, all the conditions of the Theorem (3.4) are satisfied and we note that $2 \in X$ is the unique common fixed point of $A$ and $S$. (3)

Theorem 3.6. Let $A$ and $S$ be self maps of a metric space $(X, d)$ satisfying the following conditions:

(3.6.1) $[A, S]$ is non-compatible ,

(3.6.2) $[A, S]$ is faintly compatible ,

(3.6.3) $d(A x, A A y) \neq \max \{d(A x, S A y), d(S A x, A A y)\}$, whenever the right side is non-zero , $x, y \in X$,

(3.6.4) $[A, S]$ is reciprocal continuous.

Then $A$ and $S$ have a unique common fixed point.

Proof. As $[A, S]$ is non-compatible, we get a sequence $\left\{x_{n}\right\}$ in $X$ such that $A x_{n} \rightarrow t, S x_{n} \rightarrow t$, for some $t \in X$ and $\lim _{n \rightarrow \infty} d\left(A S x_{n}, S A x_{n}\right)$ is either non-zero or non-existent. As $[A, S]$ is faintly compatible and $\lim _{n \rightarrow \infty} A x_{n}=$ $\lim _{n \rightarrow \infty} S x_{n}=t$, there is a sequence $\left\{z_{n}\right\}$ in $X$ satisfying $\lim _{n \rightarrow \infty} A z_{n}=\lim _{n \rightarrow \infty} S z_{n}=v$, for some $v \in X$, and $\lim _{n \rightarrow \infty} d\left(A S z_{n}, S A z_{n}\right)=0$. As $[A, S]$ is reciprocal continuous, we get $\lim _{n \rightarrow \infty} A S z_{n}=A v, \lim _{n \rightarrow \infty} S A z_{n}=S v$. These give $A v=S v$. In view of (3.6.2), we get $A S v=S A v$. Hence we get, $A A v=A S v=S A v=S S v$. If $A v \neq$ $A A v$, we get by (3.6.3),$d(A v, A A v) \neq \max \{d(A v, S A v), d(S A v, A A v)\}=d(A v, S A v)=d(A v, A A v) \neq 0$, which is a contradiction and so $A v=A A v=S A v$. Therefore , $A v$ is a common fixed point of $A$ and $S$. If $\alpha, \beta \in X$ are common fixed points of $A$ and $S$ and $\alpha \neq \beta$, we would get $d(A \alpha, A A \beta) \neq \max \{d(A \alpha, S A \beta), d(S A \alpha, A A \beta)\}$, i.e. , $d(\alpha, \beta) \neq d(\alpha, \beta)$, which is a contradiction and so $\alpha=\beta$. Hence the common fixed point is unique. (3)

Taking $x=y$ in (3.6.3), we get the following result, which is a generalization of a result due to Bisht and Shahzad [2, Theorem 2.4].

Corollary 3.7. Let $A$ and $S$ be self maps of a metric space $(X, d)$ satisfying the following conditions:

(3.7.1) $[A, S]$ is non-compatible ,

(3.7.2) $[A, S]$ is faintly compatible ,

(3.7.3) $d(A x, A A x) \neq \max \{d(A x, S A x), d(S A x, A A x)\}$, whenever the right side is non-zero , $x, y \in X$,

(3.7.4) $[A, S]$ is reciprocal continuous.

Then $A$ and $S$ have a common fixed point.

Proof. The proof is the same as the proof of the Theorem (3.6) without the uniqueness part. (3)

We now illustrate the Theorem (3.6) by an example below.

Example 3.8. Let $X=[2, \infty[$ and $d$ be the usual metric on $X$. Let $A, S$ be self maps of $X$ given by $A x=2$, if $2 \leq x \leq 5, A x=8$, if $x>5 . S x=2$, if $2 \leq x \leq 5, S x=x+3$, if $x>5$.

(i) Let $x_{n}=5+\frac{1}{n} \in X$. Now, $A x_{n} \rightarrow 8, S x_{n} \rightarrow 8$ and $A S x_{n} \rightarrow 8, S A x_{n} \rightarrow 11$. 
Hence, $\left.d\left(A S x_{n}, S A x_{n}\right)\right) \nrightarrow 0$ and so $[A, S]$ is non- compatible.

(ii) With $x_{n}=2+\frac{1}{n} \in X$, we see that $A x_{n} \rightarrow 2, S x_{n} \rightarrow 2$ and $A S x_{n} \rightarrow 2, S A x_{n} \rightarrow 2$.

Therefore, $d\left(A S x_{n}, S A x_{n}\right) \rightarrow 0$ and so $[A, S]$ is conditionally compatible. Also for $x \in X$,

$A x=S x$ implies $2 \leq x \leq 5$ and $A S x=S A x$. Therefore, $[A, S]$ is faintly compatible.

(iii) We get $A A x=A x$ for every $x \in X$. $S A x=2$ if $2 \leq x \leq 5$, $S A x=11$, if $x>5$.

For $2 \leq x, y \leq 5, \max \{d(A x, S A y), d(S A x, A A y)\}=0$.

For $x, y>5, d(A x, A A y)=0 \neq \max \{d(A x, S A y), d(S A x, A A y)\}=3$.

For $\quad(2 \leq x \leq 5, y>5)$ or $\quad(x>5,2 \leq y \leq 5), \quad d(A x, A A y)=6 \neq \max \{d(A x, S A y), d(S A x, A A y)\}=9$.

Therefore, the condition (3.6.3) is satisfied.

(iv) Let $x_{n} \in X$ be such that $A x_{n} \rightarrow z, S x_{n} \rightarrow z$ in $X$. Then $z=2,2 \leq x_{n} \leq 5$ and

$A S x_{n} \rightarrow A z, S A x_{n} \rightarrow S z$. Therefore, $[A, S]$ is reciprocal continuous.

Therefore, all the conditions of the Theorem (3.6) are satisfied and we note that $2 \in X$ is the unique common fixed point of $A$ and $S$. (3)

Remark 3.9. The above Theorems $(3.1,3.4,3.6)$ remain true with proofs unchanged if we replace the condition " $[A, S]$ is non-compatible " by a weaker condition " $[A, S]$ satisfy the property $E . A$."

Remark 3.10. The Theorem (3.6) remains true if we replace the condition (3.6.3) by

$" d(S x, S S y) \neq \max \{d(S x, A S y), d(A S x, S S y)\}$, whenever the right side is non-zero, $x \in X "$.

Remark 3.11. Fixed points of Corollary (3.7) need not be unique. For example, let us consider the usual metric space $(X, d)$, where $X=[0,2]$. Let $A$ and $S$ be the self maps of $X$ given by

$$
A x=\left\{\begin{array}{l}
x, \text { if } 0 \leq x \leq 1 \\
2, \text { if } 1<x \leq 2 .
\end{array} \text { and } S x=\left\{\begin{array}{c}
2-x, \text { if } 0 \leq x \leq 1 . \\
2, \text { if } 1<x \leq 2
\end{array}\right.\right.
$$

Then $A$ and $S$ satisfy all the conditions of the Corollary (3.7) and 1,2 $\in X$ are distinct common fixed points of $A$ and $S$. It may be verified that $[A, S]$ is non-compatible (consider the sequence $x_{n}=1-\frac{1}{n}$ ). Moreover $[A, S]$ is faintly compatible and reciprocal continuous. Furthermore, $A x=A A x$ and so the condition (3.7.3) is satisfied. (3)

\section{ACKNOWLEDGEMENTS}

The first author gratefully acknowledges the constant support and encouragement he has received from Dr. N. R. Das of Gauhati University, while preparing the paper.

\section{REFERENCES}

[1] M. Aamri , D.El Moutawakil, some new common fixed point theorems under strict contractive conditions , J. Math. Anal. Appl. , 270(2002), 181-188.

[2] R. K. Bisht, N. Shahzad, Faintly compatible mappings and common fixed points, Fixed point theory and applications , $2013,2013: 156$.

[3] R. K. Bisht, Common fixed points of generalized Meir-Keeler type condition and non-expansive mappings, Int. J. Math. Math. Sci. 2012, Article ID 786814(2012).

[4] N. R. Das , M. L. Saha, Some fixed point theorems in fuzzy 2- metric spaces, J. Fuzzy Math., 21(4) (2013) $753-758$.

[5] N. R. Das, M. L. Saha, On fixed points in fuzzy metric spaces, Ann. Fuzzy Math. Inform.7(2) (2014) 313-318

[6] G. Jungck, Commuting mappings and common fixed points , Amer. Math. Month. , 83(1976) , 261-263.

[7] G. Jungck, Compatible mappings and common fixed points, Internat. J. Math. Math. Sci. , 9(4) (1986) ,771-779.

[8] G. Jungck, Common fixed points for commuting and compatible maps on compacta, Proc. Amer. Math. Soc. , 103(3) , (1988) , 977-983.

[9] G. Jungck, Compatible mappings and common fixed points (2), Internat. J. Math. Math. Sci. ,11(2) , (1988) , $285-288$.

[10] G. Jungck, Common fixed points for non continuous non self mappings on non metric spaces, Far East J. Math. Sci. , 4(1996) , 199-215. 
American Research Journal of Mathematics, Volume 1, Issue 2, April 2015

ISSN 2378-704X

[11] R. P. Pant , Common fixed points of non-commutating maps , J. Math. Anal. Appl. ,188 (1994) 436-440.

[12] R. P. Pant, V. Pant, Common fixed points under strict contractive conditions , J. Math. Anal.Appl. , 248(2000) , $327-332$.

[13] R. P. Pant, Common fixed point theorems for contractive maps , J. Math. Anal. Appl. , 226 (1998) 251-258.

[14] R. P. Pant , A common fixed point theorem under a new condition, Indian J. Pure Appl. Math. 30(2) (1999) , $147-152$.

[15] R. P. Pant, R. K. Bisht, Occasionally weakly compatible mappings and fixed points, Bull. Belg. Math. Soc. Simon Stevin , 19 (2012), 655-661.

[16] S.Sessa, On weak commutativity condition of mappings in fixed point consideration, Publ. Inst. Math. , 32 (1982) , $149-153$. 